\title{
Serum omentin-1 level in patients with benign prostatic hyperplasia
}

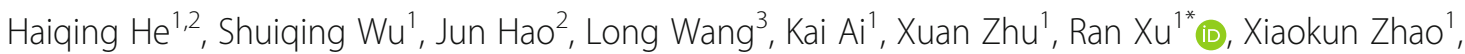 \\ Yinhuai Wang ${ }^{1}$ and Zhaohui Zhong ${ }^{1}$
}

\begin{abstract}
Backgroud: To evaluate the relationship between omentin-1 and benign prostatic hyperplasia (BPH). BPH is the most common urological disease in elderly men worldwide. Lower serum omentin-1 levels were reported to be negatively associated with the incidence of inflammation, diabetes, obesity and metabolic syndrome, which all play a role in the development of $\mathrm{BPH}$. To the best of our knowledge, the relationship between omentin-1 and BPH has not been investigated previously.
\end{abstract}

Methods: A total of 70 males participated in this study, including forty patients diagnosed with BPH and thirty healthy males. The anthropometric measurements and the biochemical parameters were measured in this study. We evaluated serum omentin-1 levels and the correlation with those data. We also test the gene expression of IL-8, IL-18 in BPH group using the TURP tissues.

Results: The serum omentin-1 levels were lower in the BPH patients than in the control group ( $27.95 \pm 4.18$ versus $32.03 \pm 5.46, p<0.001)$. The general characteristics and biochemical parameters were investigated, and a negative correlation was found between serum omentin-1 levels and BMI in the BPH group ( $r=-0.391, p=0.013)$ as well as the whole group $(r=-0.457, p<0.001)$. Multiple-factor binary regression analysis revealed that serum omentin1 was a protective factor of BPH development. Furthermore, lower serum omentin-1 levels were associated with higher mRNA expression of IL-8 or IL-18 in the BPH group.

Conclusion: Omentin-1 may suppress the development of BPH and Lower serum omentin-1 levels in BPH patients might associated with higher prostate volume and higher IL-8 and IL-18 expression levels in their prostatic cells.

Keywords: Benign prostatic hyperplasia (BPH), Omentin/Omentin-1, Adipokine, Prostate volume, Interleukin-8, Interleukin-18

\section{Backgroud}

Benign prostatic hyperplasia $(\mathrm{BPH})$ is the most common urological disease in elderly men worldwide. BPH causes prostate enlargement and is associated with bladder outlet obstruction and various lower urinary tract symptoms (LUTS) [1]. BPH can also cause the deterioration of urinary function and quality of life and increase the

\footnotetext{
*Correspondence: xuran@csu.edu.cn

${ }^{1}$ Department of Urology, The Second Xiangya Hospital, Central South University, Changsha 410011, Hunan, China

Full list of author information is available at the end of the article
}

risk of both urinary tract infection (UTI) and acute urinary retention, which requires surgical attention. Moreover, the medical costs for managing $\mathrm{BPH}$ are high, despite the fact that many men with $\mathrm{BPH}$ remained underdiagnosed and undertreated.

Although the mechanisms are not fully clear, metabolic status, inflammation, and hormonal status may contribute to the pathogenesis of BPH. Previous studies demonstrated evidence of strong correlations between metabolic syndrome and chronic inflammation in $\mathrm{BPH}$ pathogenesis and progression [2]. 
Omentin was primarily identified from cDNA library of human omental adipose tissue [3]. Omentin-1 (Gene name ITLN1), which is an isoform of omentin, shows its abundance in human plasma. Decreased serum omentin-1 levels are detected in patients with endocrine diseases such as type 2 diabetes and obesity [4]. Meanwhile, it was proved that reduced omentin-1 level plays pivotal role in the causation of inflammatory diseases [5].

Previous studies on the association of $\mathrm{BPH}$ and adipokines (e.g., C-peptide, leptin and adiponectin) have reported conflicting results [6-8]. To the best of our knowledge, there are no reports of a relationship between omentin and $\mathrm{BPH}$. Therefore, this matched case-control study investigated the serum omentin concentrations in patients with $\mathrm{BPH}$.

\section{Methods}

\section{Subjects}

This study was approved by the Ethics Committee of the Second Xiangya Hospital of Central South University. A total of 70 males seen from January 2017 to January 2018 gave informed consent before participating in this study. Forty patients diagnosed with BPH who needed transurethral resection of the prostate (TURP) and thirty healthy males matched by age were assigned into two groups: the $\mathrm{BPH}$ group and the control group (CG). $\mathrm{BPH}$ was diagnosed based on clinical findings, rectal examination, ultrasound and confirmed by histopathological results. The healthy males were defined by ultrasound and International Prostate Symptom Score (IPSS). The prostate size of CG measured by ultrasound is less than $4 \mathrm{~cm} \times 3 \mathrm{~cm} \times 2 \mathrm{~cm}$ and IPSS is less than 7 . The PSA values of all these men were less than $4 \mathrm{ng} / \mathrm{mL}$.

\section{Methods}

The anthropometric measurements obtained in this study included height, weight, systolic blood pressure (SBP), diastolic blood pressure (DBP), and waist circumference (WC). Body mass index (BMI) was calculated as the body weight divided by the height squared $(\mathrm{kg} / \mathrm{m} 2)$. We measured the WC at the umbilicus level. Biochemical parameters were measured in venous blood samples taken after at least a 10-h fast. Fasting blood samples were obtained to analyze blood urea nitrogen (BUN), triglyceride (TG), total cholesterol (TC), high-density lipoprotein (HDL), creatinine, prostate-specific antigen (PSA) and glucose levels using standard methods. The estimated glomerular filtration rates (eGFRs) were assessed using the following equation: eGFR $=\alpha \times($ $\mathrm{SC} \div 0.9)-\beta \times 0.993$ age [Note: $\mathrm{SC}$ is the serum creatinine level in $\mathrm{mg} / \mathrm{ml} \alpha$ is 128 , and $\beta$ is 0.015 for $\mathrm{SC} \leq 0.9 \mathrm{mg} /$ $\mathrm{dl}$ or $79.56 \mu \mathrm{mol} / \mathrm{l}$; otherwise, $\alpha$ is 119 , and $\beta$ is 0.688 ]. Prostate size was measured by ultrasound and the prostate volume (PV) was calculated from transverse images by using the prolate-ellipsoid formula: $0.524 \times$ height $x$ width $\times$ length [9].

\section{Omentin-1 measurement}

Venous blood was collected after fasting followed with centrifugation of $4000 \mathrm{rpm}$ at room temperature. Afterward, enzyme-linked immunosorbent assay (ELISA) was performed to determine concentration of omentin-1 in human serum (Apotech, Inc.).

Quantification of gene expression in BPH tissues by quantitative real-time PCR.

Total RNA was extracted using an RNAsimple Total RNA Kit (TIANGEN Biotech) and miRcute miRNA Isolation Kit (TIANGEN Biotech) and reverse transcribed using a RevertAid First Strand cDNA Synthesis Kit (Thermo Fisher). Quantitative RT-PCR was performed using SuperReal PreMix Plus (TIANGEN Biotech) and the following primers:

IL-8:

Fwd: CCAGGAAGAAACCACCGGA

Rev: GAAATCAGGAAGGCTGCCAAG

IL-18:

Fwd: AAAACCTGGAATCAGATTACTTTGG

Rev: TCCGGGGTGCATTATCTCTA

$\beta$-actin:

Fwd: TTCCTTCCTGGGCATGGAGTC

Rev: TCTTCATTGTGCTGGGTGCC

Specific gene expression was quantified using the 2$\Delta \Delta \mathrm{CT}$ method. Gene expression normalization was performed using $\beta$-actin as a reference gene.

\section{Statistical analysis}

SPSS 22.0 software was used for the statistical analyses. The results are presented as the mean \pm standard deviation (SD). Student's t-test was used to determine the significance between two groups. Spearman's rank correlation coefficient analyses ( $r$ ) and the coefficient of determination $\left(\mathrm{r}^{2}\right)$ were conducted to test for associations between omentin-1 levels and general clinical characteristics, biochemical parameters and relative gene expression. The influential factors for omentin-1 levels were identified using multiple-factor binary logistic regression analysis for meaningful clinical characteristics and biochemical parameters. Finally, the influential factors for $\mathrm{BPH}$ development were identified using multiple-factor binary logistic regression analysis for meaningful clinical characteristics and biochemical parameters. $P<0.05$ was considered statistically significant, * indicates $p<0.05$, ** indicates $p<0.01,{ }^{* * *}$ indicates $p<0.001$.

\section{Results}

The general characteristics and biochemical parameters of the two groups are listed in Table 1. There were no statistically significant differences in age, WC, fasting 
Table 1 Comparison of the general characteristics and biochemical parameters

\begin{tabular}{llll}
\hline & $\begin{array}{l}\text { BPH } \\
(n=40)\end{array}$ & $\begin{array}{l}\text { CG } \\
(n=30)\end{array}$ & $p$ \\
\hline Age (years) & $59.15 \pm 7.382$ & $59.07 \pm 7.593$ & 0.963 \\
BMI (kg/m $\left.{ }^{2}\right)$ & $26.20 \pm 3.48$ & $22.56 \pm 3.01$ & $<\mathbf{0 . 0 0 1}$ \\
SBP $(\mathrm{mmHg})$ & $126.6 \pm 10.8$ & $124.5 \pm 11.8$ & 0.706 \\
DBP $(\mathrm{mmHg})$ & $76.1 \pm 8.3$ & $72 \pm 9.2$ & 0.059 \\
Waist circumference $(\mathrm{cm})$ & $90.33 \pm 14.06$ & $88.93 \pm 12.89$ & 0.671 \\
TG (mmol/L) & $1.81 \pm 0.26$ & $1.71 \pm 0.18$ & 0.091 \\
TC (mmol/L) & $4.56 \pm 0.86$ & $4.14 \pm 1.01$ & 0.073 \\
HDL (mmol/L) & $1.22 \pm 0.31$ & $1.18 \pm 0.24$ & 0.561 \\
Fasting glucose $(\mathrm{mmol} / \mathrm{L})$ & $4.76 \pm 0.96$ & $4.34 \pm 0.94$ & 0.076 \\
BUN (mmol/L) & $5.40 \pm 1.57$ & $4.80 \pm 1.12$ & 0.084 \\
eGFR (mL/min/1.73 m $\left.{ }^{2}\right)$ & $76.18 \pm 8.37$ & $79.97 \pm 7.64$ & 0.055 \\
Creatinine $(\mu \mathrm{mol} / \mathrm{L})$ & $81.46 \pm 17.26$ & $72.92 \pm 17.63$ & $\mathbf{0 . 0 4 7}$ \\
PSA (ng/mL) & $2.29 \pm 0.89$ & $1.72 \pm 0.73$ & $\mathbf{0 . 0 0 5}$ \\
Omentin-1 (ng/mL) & $27.31 \pm 4.56$ & $33.47 \pm 4.92$ & $<\mathbf{0 . 0 0 1}$ \\
Prostate volume $(\mathrm{mL})$ & $62.00 \pm 30.26$ & $10.64 \pm 1.29$ & $<\mathbf{0 . 0 0 1}$ \\
\hline
\end{tabular}

glucose, SBP, DBP, TG, TC, HDL, fasting glucose, BUN and eGFR between the two groups. BMI, BUN, creatinine, PSA levels were significantly higher in the $\mathrm{BPH}$ group than in the CG. The omentin-1 levels were lower in the BPH group than in the CG (Fig. 1a).

Multiple-factor binary regression analysis was performed to reveal the risk factors for $\mathrm{BPH}$ development from various meaningful clinical characteristics and biochemical parameters, including BMI, creatinine, PSA and omentin-1 (Table 2). Among these data, BMI and omentin-1 level were significantly associated with the $\mathrm{BPH}$ development.

Correlations between the serum omentin-1 level and the biochemical properties in the BPH, CG and whole group were determined with Spearman's rank correlation coefficient analysis. It was suggestive that serum omentin-1 levels have little correlation with age, SBP, DBP, WC, TG,TC,HDL, fasting glucose, BUN, eGFR or creatinine while negative correlations were found between serum omentin-1 levels and BMI in the BPH group $(\mathrm{r}=-0.391, p=0.013)$ as well as the whole group $(\mathrm{r}=-0.457, p<0.001)$ (Supplementary Table 1$)$.

The BPH patients have been divided in two group according to the IPSS level, IPSS 8-19 and IPSS 20-35. The serum omentin-1 level in these two group showed no significantly difference (Fig. 1b). And also, there was no significant correlation between serum omentin-1 and IPSS in either BPH group or CG (Fig. 2a, b). Alternative, serum omentin-1 level showed significant negative correlations with prostate volume in BPH group (Supplementary Table 2, Fig. 2c, d).

Interleukin-8 (IL-8) and interleukin-18 (IL-18) are two cytokines secreted mainly by prostatic cells and functionally related to Omentin-1 (Supplementary Figure 1) [10-13]. It has been shown that the mRNA expression of these two cytokines was significantly higher in $\mathrm{BPH}$ patients with LUTS [11-13]. Thus, in this study, we also checked the expression levels of IL-8 and IL-18 in our $\mathrm{BPH}$ patient samples. We found that the mRNA expression levels of IL- 8 and IL-18 were significantly higher in the low serum omentin-1 group (levels lower than the median) (Fig. 3a, b). Moreover, there were significant negative correlations between serum omentin-1 levels

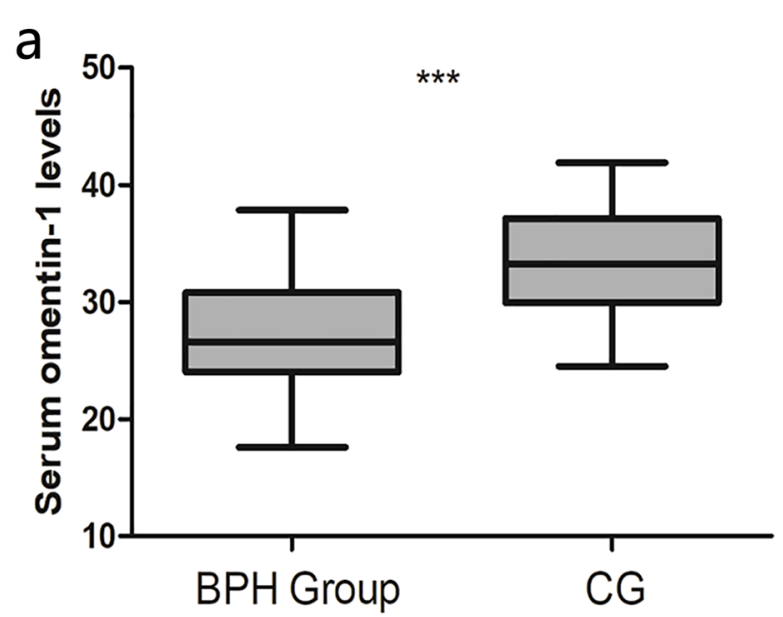

b

Fig. 1 Serum omentin-1 levels. a. Serum omentin-1 levels in the BPH groups and CG. The concentrations of omentin-1 in human serum were determined using an enzyme-linked immunosorbent assay (ELISA) kit. b. The serum omentin-1 level in different IPSS level in BPH group. The BPH patients have been divided in two group according to the IPSS level. IPSS 8-19 indicates Level 2. IPSS 20-35 indicates level 3. The Vertical histograms show means \pm SD 
Table 2 Multiple-factor binary logistic regression analysis

\begin{tabular}{llllll}
\hline Variables & B value & SE value & OR value & $95 \% \mathrm{Cl}$ & $P$ value \\
\hline BMI $\left(\mathrm{kg} / \mathrm{m}^{2}\right)$ & 0.242 & 0.120 & 1.274 & $1.008-1.611$ & $\mathbf{0 . 0 4 3}$ \\
PSA $(\mathrm{ng} / \mathrm{mL})$ & 0.457 & 0.390 & 1.579 & $0.720-3.395$ & 0.242 \\
Creatinine $(\mu \mathrm{mol} / \mathrm{L})$ & 0.036 & 0.019 & 1.036 & $0.998-1.077$ & 0.065 \\
Omentin-1 $(\mathrm{ng} / \mathrm{mL})$ & -0.206 & 0.077 & 0.813 & $0.699-0.946$ & $\mathbf{0 . 0 0 8}$
\end{tabular}

SE standard error, OR odds ratio, $\mathrm{Cl}$ confidence interval

and IL- 8 and IL-18 mRNA expression in the BPH group (Supplementary Table 2, Fig. 3c, d).

\section{Discussion}

With the changing demographic profile and an increasingly aging population in almost all societies, it is inevitable that the prevalence of $\mathrm{BPH}$ will increase.

Obesity is often comorbid with BPH. Body weight, $\mathrm{BMI}$, and WC have been reported to be positively associated with prostate volume. Moreover, obesity was also associated with an increased risk of symptomatic $\mathrm{BPH}$ in several different international studies $[14,15]$. In the present study, WC was greater in patients in the $\mathrm{BPH}$ group than those in the CG, but the difference was not statistically significant. BMI was significantly greater for the patients in the BPH group than for those in the CG, which suggests that increased body weight could be a factor in the etiology of $\mathrm{BPH}$.

Several studies were done to examine the influence of diabetes using more objective measures of $\mathrm{BPH}$, specifically prostate gland size. Increased prostate size and $\mathrm{BPH}$ surgery have strong association with the diagnosis of diabetes and increased serum insulin fasting plasma glucose level [16, 17]. In our study, glucose levels were not statistically significantly higher in the BPH group.

It is likely that inflammation plays a role in the development and progression of $\mathrm{BPH}[1,18]$ because inflammatory cytokines are overexpressed in BPH tissues [19]. It remains unclear how inflammation mediates $\mathrm{BPH}$. Obesity is considered a hormone-regulated disease when the secretion of pro-inflammatory cytokines and chemokine can promote the enlargement of adipocytes and at the mean time, influence prostate health [20]. These active hormones are known as adipokines, such as adiponectin. High adiponectin concentrations were associated with a reduced risk of symptomatic BPH [6].

Omentin is an anti-inflammatory adipokine in human with a higher expression in visceral adipose tissue than those found subcutaneously [21]. Omentin-1 is the major circulating form of omentin. Omentin showed a strong negative correlation with the incidence of inflammation, diabetes, obesity and metabolic syndrome [4], a
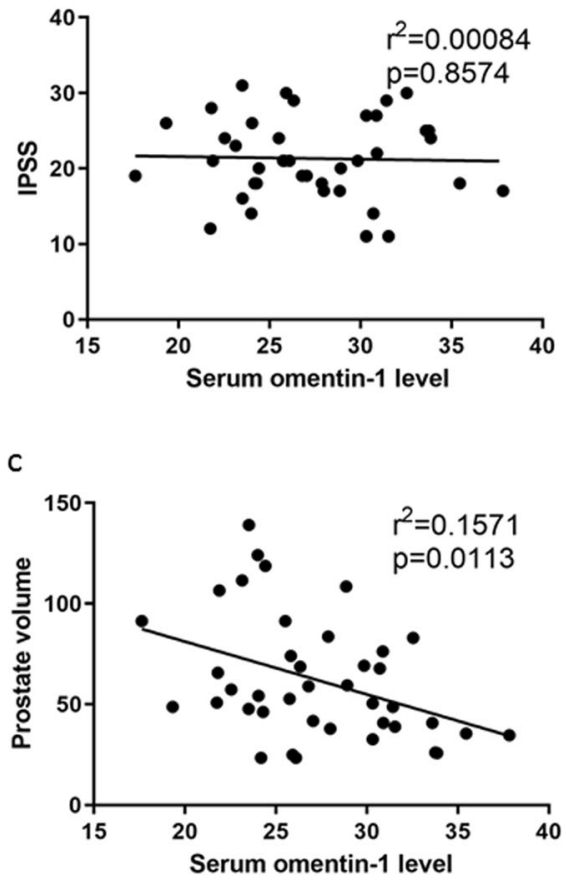

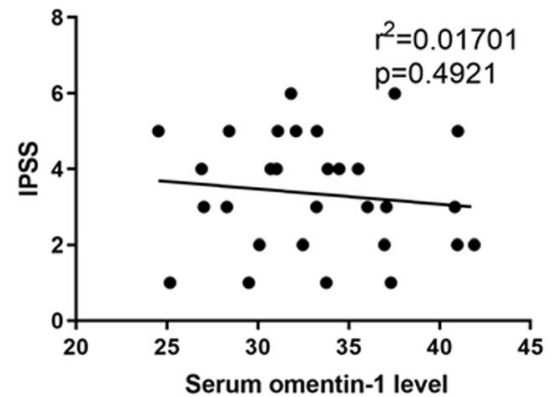

d

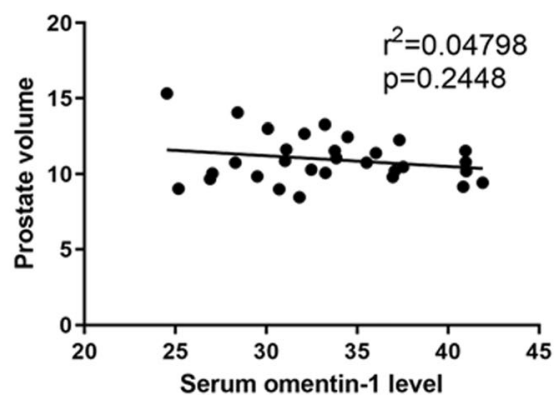

Fig. 2 The correlation between serum omentin-1 and IPSS/Prostate volume. a. The coefficient of determination for IPSS and serum omentin-1 level in BPH group was established. b. The coefficient of determination for for IPSS and serum omentin-1 level in CG was established. c. The coefficient of determination for prostate volume and serum omentin-1 level in BPH group was established. $\mathbf{d}$. The coefficient of determination for prostate volume and serum omentin-1 level in CG was established. The trend line is shown 
a

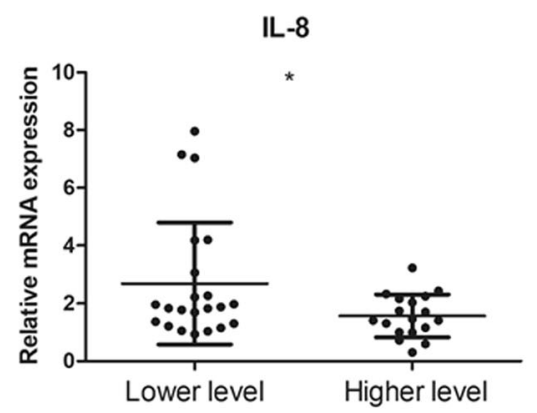

C

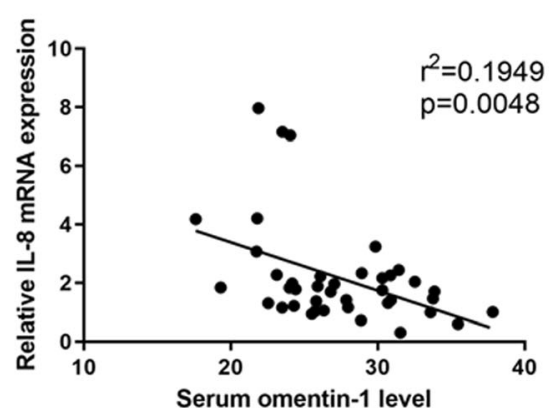

b

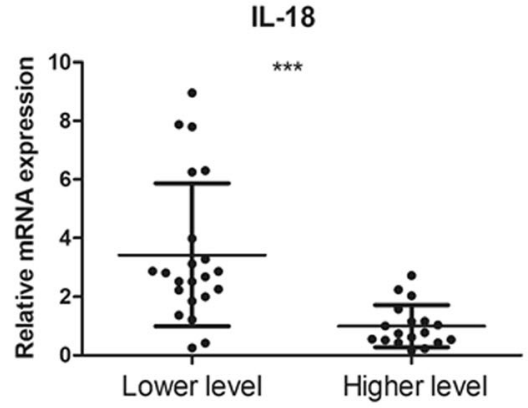

d

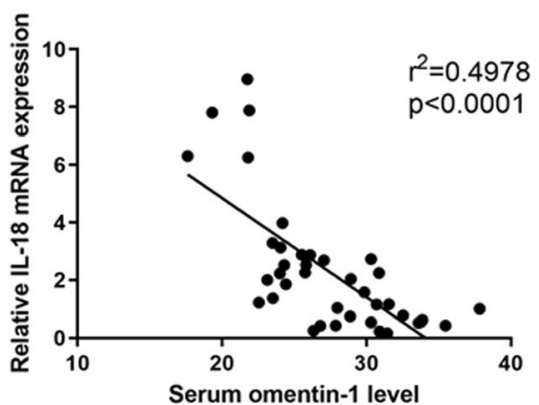

Fig. 3 Relative gene mRNA expression according to omentin-1 Levels and their correlation. $\mathbf{a}, \mathbf{b}$. IL-8 and IL-18 mRNA expressions in clinical BPH specimen were determined by qRT-PCR. BPH patients were divided into two groups based on their serum omentin-1 levels. Results are presented as means $\pm \mathrm{SD}$. Specific gene expression was quantified using the $2-\Delta \Delta C T$ method. Gene expression normalization was performed using $\beta$-actin as a reference gene. $\mathbf{c}$. The coefficient of determination for IL-8 mRNA expression and serum omentin-1 level was established. $\mathbf{d}$. The coefficient of determination for IL-18 mRNA expression and serum omentin-1 level was established. The trend line is shown

which all contribute to $\mathrm{BPH}$ progression. Decreased omentin-1 levels may play a role in the development of insulin resistance, type 2 diabetes mellitus and particularly obesity [22]. Through homeostasis model assessment, it was demonstrated that omentin-1 level is negatively correlated with BMI, WC and insulin resistance while it is positively associated HDL and adiponectin level [23]. A previous study showed that omentin-1 could downregulate pro-inflammatory cytokines to prevent inflammation-induced osteoporosis [5]. Di has reported that omentin suppresses pulmonary inflammation and promotes the endothelial barrier to prevent lipopolysaccharide (LPS)-induced acute respiratory distress syndrome (ARDS) [24]. Yao found that during prostatic hyperplasia, LPS/Toll-like receptor 4 signaling could upregulate transforming growth factor- $\beta$ (TGF- $\beta$ ), which plays a role in the pathogenesis of $\mathrm{BPH}[25,26]$. Patients diagnosed with inflammatory bowel diseases such as Crohn's disease or ulcerative colitis have lower level of circulating omentin-1 [27]. Uyeturk reported that serum omentin-1 levels were significantly higher in prostate cancer $(\mathrm{PCa})$ patients than in $\mathrm{BPH}$ patients [28]. However, serum omentin-1 levels have not been previously examined in BPH patients and healthy people. In our present study, omentin-1 levels were dysregulated in patients in the BPH group compared to those in the CG. PSA levels were significantly different between the two groups but were negatively correlated with omentin-1 in the whole group. In addition, serum omentin-1 levels were negatively correlated with $\mathrm{BMI}$ in the $\mathrm{BPH}$ group and in the whole group. Serum omentin-1 level also showed significant negative correlation with prostate volume in $\mathrm{BPH}$ group. Furthermore, we found that omentin-1 might be a protective factor of $\mathrm{BPH}$ development.

In patients with acute myocardial infarction, omentin1 negatively correlated with IL-18, and IL- 18 remained an independent determinant of omentin-1 levels [29]. In research on obese people who participated in training experiments, serum omentin-1 levels decreased significantly, and IL-18 levels increased significantly after detraining [30]. These studies showed that serum omentin- 1 levels may negatively correlate with IL-18 in BPH tissues. IL-18 could be secreted by prostatic epithelial cells and respond to several inflammatory stimuli [31]. IL-18 itself directly influences prostatic stromal cell proliferation [12]. Similarly, our results suggested that in the $\mathrm{BPH}$ group, serum omentin-1 levels were significantly negatively correlated with IL-18 expression in prostatic cells. IL-8, produced by BPH prostate epithelial cells as a reliable biomarker of inflammation in $\mathrm{BPH}$, 
can promote the proliferation of non-senescent epithelial and stromal cells and contributes to the increased tissue growth in BPH $[11,13]$. IL-8 expression was also significantly negatively correlated with serum omentin-1 levels in our investigation, which supports the findings that omentin-1 could reduce IL-8 production in kidney tissue [32]. From all these data, what can be hypothesized is that men with higher BMI might show lower serum omentin-1 level, thereby contribute to the development of $\mathrm{BPH}$ via facilitating the activation of prostatic inflammation.

\section{Conclusion}

In summary, we found that serum omentin-1 levels were decreased in patients with BPH. Omentin-1 may suppress the development of $\mathrm{BPH}$ and Lower serum omentin-1 levels in BPH patients might associated with higher prostate volume and higher IL- 8 and IL-18 expression levels in their prostatic cells. Further studies establishing the mechanism underlying this phenomenon would allow better management of BPH development.

\section{Supplementary information}

Supplementary information accompanies this paper at https://doi.org/10. 1186/s12894-020-00623-4.

Additional file 1: Figure S1. Functional protein association networks of Omentin-1 (ITLN1). The functional protein association networks of Omentin-1 (Gene name: ITLN1) was shown. Cytokines such as Interleukin18 (IL18) were reported to be closely linked to ITLN1.

Additional file 2: Table S1. Spearman's rank correlation coefficient analysis of serum omentin-1 levels with the general clinical characteristics and biochemical parameters.

Additional file 3: Table S2. Spearman's rank correlation coefficient analysis of serum omentin-1 levels with the general clinical characteristics and biochemical parameters.

\section{Abbreviations}

$\mathrm{BPH}$ : benign prostatic hyperplasia; BMI: Body mass index; SBP: Systolic blood pressure; DBP: Diastolic blood pressure; WC: Waist circumference; BUN: Blood urea nitrogen; TG: Triglyceride; TC: Total cholesterol; HDL: High-density lipoprotein; PSA: Prostate-specific antigen; eGFRs: Estimated glomerular filtration rates; PV: Prostate volume; CG: Control group; IL-8: Interleukin-8; IL18: Interleukin-18

\section{Acknowledgements}

Not applicable.

\section{Authors' contributions}

Conception and design: R.X. Acquisition of data: H. H, S.W, K.A, X.Z1, R.X. Analysis and interpretation of data: H.H, S.W, J.H, K.A. Drafting of the manuscript: H.H, J.H, R.X. Critical revision of the manuscript for important intellectual content: H.H, J.H, X.Z2, Y.W, Z.Z. Obtaining funding: Y.W, R.X, Z.Z, L.W. Supervision: R.X, X.Z2. The authors read and approved the final manuscript.

\section{Funding}

This research was supported by the National Natural Science Foundation of China Grant 81770758 (to L.W.). Haiqing He is sponsored by the China Scholarship Council as a joint training Ph. D student at the University of British Columbia. The funder (China Scholarship Council) had no role in the study design, data collection and analysis, decision to publish, or preparation of the manuscript.

\section{Availability of data and materials}

The datasets used and/or analysed during the current study are available from the corresponding author on reasonable request.

\section{Ethics approval and consent to participate}

This study was approved by the Ethics Committee of the Second Xiangya Hospital of Central South University. A total of 70 males seen from January 2017 to January 2018 gave informed consent in written format before participating in this study.

\section{Consent for publication}

Not applicable.

\section{Competing interests}

We declare that there is no conflict of interest.

\section{Author details}

${ }^{1}$ Department of Urology, The Second Xiangya Hospital, Central South University, Changsha 410011, Hunan, China. ${ }^{2}$ Vancouver Prostate Centre, Department of Urologic Sciences, Faculty of Medicine, University of British Columbia, Vancouver, BC, Canada. ${ }^{3}$ Department of Urology, Xiangya Hospital, Central South University, Changsha 410008, Hunan, China.

Received: 30 May 2019 Accepted: 29 April 2020

Published online: 06 May 2020

References

1. Inamura S, et al. Prostatic stromal inflammation is associated with bladder outlet obstruction in patients with benign prostatic hyperplasia. Prostate. 2018;78(10):743-52.

2. De Nunzio C, Presicce F, Tubaro A. Inflammatory mediators in the development and progression of benign prostatic hyperplasia. Nat Rev Urol. 2016;13(10):613-26.

3. Yang RZ, et al. Identification of omentin as a novel depot-specific adipokine in human adipose tissue: possible role in modulating insulin action. Am J Physiol Endocrinol Metab. 2006;290(6):E1253-61.

4. Watanabe T, et al. Adipose tissue-derived Omentin-1 function and regulation. Compr Physiol. 2017;7(3):765-81.

5. Rao SS, et al. Omentin-1 prevents inflammation-induced osteoporosis by downregulating the pro-inflammatory cytokines. Bone Res. 2018;6:9.

6. Schenk JM, et al. Serum adiponectin, C-peptide and leptin and risk of symptomatic benign prostatic hyperplasia: results from the prostate Cancer prevention trial. Prostate. 2009;69(12):1303-11.

7. Grosman $\mathrm{H}$, et al. Complex relationship between sex hormones, insulin resistance and leptin in men with and without prostatic disease. Aging Male. 2016;19(1):40-5.

8. Fu S, et al. Adiponectin deficiency contributes to the development and progression of benign prostatic hyperplasia in obesity. Sci Rep. 2017;7: 43771.

9. Eri LM, et al. Accuracy and repeatability of prostate volume measurements by transrectal ultrasound. Prostate Cancer Prostatic Dis. 2002:5(4):273-8.

10. Szklarczyk D, et al. The STRING database in 2017: quality-controlled proteinprotein association networks, made broadly accessible. Nucleic Acids Res. 2017:45(D1):D362-8.

11. Castro $P$, et al. Interleukin-8 expression is increased in senescent prostatic epithelial cells and promotes the development of benign prostatic hyperplasia. Prostate. 2004;60(2):153-9.

12. Hamakawa T, et al. Interleukin-18 may lead to benign prostatic hyperplasia via thrombospondin-1 production in prostatic smooth muscle cells. Prostate. 2014;74(6):590-601.

13. Liu L, et al. Evaluation of interleukin-8 in expressed prostatic secretion as a reliable biomarker of inflammation in benign prostatic hyperplasia. Urology. 2009:74(2):340-4.

14. Dahle SE, et al. Body size and serum levels of insulin and leptin in relation to the risk of benign prostatic hyperplasia. J Urol. 2002;168(2):599-604.

15. Kristal AR, et al. Race/ethnicity, obesity, health related behaviors and the risk of symptomatic benign prostatic hyperplasia: results from the prostate cancer prevention trial. J Urol. 2007;177(4):1395-400 quiz 1591. 
16. Sarma AV, et al. Diabetes and benign prostatic hyperplasia/lower urinary tract symptoms--what do we know? J Urol. 2009;182(6 Suppl):S32-7.

17. Kim WT, et al. Prostate size correlates with fasting blood glucose in nondiabetic benign prostatic hyperplasia patients with Normal testosterone levels. J Korean Med Sci. 2011;26(9):1214-8.

18. Zlotta $A R$, et al. Prevalence of inflammation and benign prostatic hyperplasia on autopsy in Asian and Caucasian men. Eur Urol. 2014;66(4): 619-22.

19. Schenk JM, et al. Biomarkers of systemic inflammation and risk of incident, symptomatic benign prostatic hyperplasia: results from the prostate cancer prevention trial. Am J Epidemiol. 2010;171(5):571-82.

20. Di Silverio F, et al. Distribution of inflammation, pre-malignant lesions, incidental carcinoma in histologically confirmed benign prostatic hyperplasia: a retrospective analysis. Eur Urol. 2003;43(2):164-75.

21. Martinez-Garcia MA, et al. Evidence for masculinization of adipokine gene expression in visceral and subcutaneous adipose tissue of obese women with polycystic ovary syndrome (PCOS). J Clin Endocrinol Metab. 2013;98(2): E388-96.

22. Zhang $\mathrm{Q}$, et al. Changes of serum omentin-1 levels in normal subjects, type 2 diabetes and type 2 diabetes with overweight and obesity in Chinese adults. Ann Endocrinol (Paris). 2014;75(3):171-5.

23. de Souza Batista CM, et al. Omentin plasma levels and gene expression are decreased in obesity. Diabetes. 2007;56(6):1655-61.

24. Qi D, et al. Omentin protects against LPS-induced ARDS through suppressing pulmonary inflammation and promoting endothelial barrier via an Akt/eNOS-dependent mechanism. Cell Death Dis. 2016;7(9):e2360.

25. He Y, et al. LPS/TLR4 signaling enhances TGF-beta response through Downregulating BAMBI during prostatic hyperplasia. Sci Rep. 2016;6:27051.

26. Wang $L$, et al. Aberrant transforming growth factor-beta activation recruits Mesenchymal stem cells during prostatic hyperplasia. Stem Cells Transl Med. 2017;6(2):394-404

27. Yin J, et al. Decreased levels of serum omentin-1 in patients with inflammatory bowel disease. Med Sci Monit. 2015;21:118-22.

28. Uyeturk $\mathrm{U}$, et al. Serum omentin level in patients with prostate cancer. Med Oncol. 2014;31(4):923.

29. Kadoglou NP, et al. Serum levels of novel adipokines, omentin-1 and chemerin, in patients with acute myocardial infarction: KOZANI STUDY. J Cardiovasc Med (Hagerstown). 2015;16(5):341-6.

30. Nikseresht M, Hafezi Ahmadi MR, Hedayati M. Detraining-induced alterations in adipokines and cardiometabolic risk factors after nonlinear periodized resistance and aerobic interval training in obese men. Appl Physiol Nutr Metab. 2016;41(10):1018-25.

31. Wang $X$, et al. Increased infiltrated macrophages in benign prostatic hyperplasia (BPH): role of stromal androgen receptor in macrophageinduced prostate stromal cell proliferation. J Biol Chem. 2012;287(22):1837685.

32. Song J, et al. Omentin-1 protects renal function of mice with type 2 diabetic nephropathy via regulating miR-27a-Nrf2/Keap1 axis. Biomed Pharmacother. 2018;107:440-6.

\section{Publisher's Note}

Springer Nature remains neutral with regard to jurisdictional claims in published maps and institutional affiliations.

Ready to submit your research? Choose BMC and benefit from:

- fast, convenient online submission

- thorough peer review by experienced researchers in your field

- rapid publication on acceptance

- support for research data, including large and complex data types

- gold Open Access which fosters wider collaboration and increased citations

- maximum visibility for your research: over $100 \mathrm{M}$ website views per year

At $\mathrm{BMC}$, research is always in progress.

Learn more biomedcentral.com/submissions 